\title{
Comprehensive Modeling of Diffused Quantum-Well Vertical-Cavity Surface-Emitting Lasers
}

\author{
W. M. Man and S.-F. Yu
}

\begin{abstract}
A numerical model for investigating the thermal, electrical, and optical characteristics of vertical-cavity surfaceemitting lasers (VCSEL's) with a diffused quantum-well (QW) structure is presented. In the model, the quasi-three-dimensional (quasi-3-D) distribution of temperature, voltage and optical fields as well as the quasi-two-dimensional (quasi-2-D) diffusion and recombination of carrier concentration inside the $Q W$ active layer are calculated in a self-consistent manner. In addition, the quasi-3-D distribution of implanted ions before and after thermal annealing are computed. The variation of electrical conductivity and absorption loss as well as the influence of impurity induced compositional disordering on the optical gain and refractive index of the QW active layer are also taken into consideration. Using this model, the steady-state characteristics of diffused QW VCSEL's are studied theoretically. It is shown that significant improvement of stable single-mode operation can be obtained using diffused QW structure.
\end{abstract}

Index Terms - Interdiffusion, laser modeling, quantum-well semiconductor lasers, vertical-cavity surface-emitting lasers.

\section{INTRODUCTION}

I MPURITY-induced compositional disordering in quantumwell $(\mathrm{QW})$ materials becomes a very important emerging technology for the fabrication of photonic and optoelectronic devices. This is because selective implantation or diffusion can be achieved by masking into the desired regions of a QW structure, a refractive index step can be obtained between the as-grown and disordered regions due to the modification of QW's bandgap. Thus, this technology enables a planar process to be used in the fabrication of three-dimensional (3-D) devices such as optical waveguides, modulators and lasers on a single substrate and makes optoelectronics integration a reality [1].

Recently, Fabry-Pérot and distributed feedback (DFB) semiconductor lasers with diffused QW's structures have been proposed for high-power single-longitudinal-mode operation [2], [3]. This is because the longitudinal distribution of refractive index inside the laser cavities can be varied by the interdiffusion technique. Hence, some undesired characteristics of semiconductor lasers such as spatial-hole burning (SHB) of carrier concentration, excitation of side mode and broadening of linewidth can be minimized. Using the same technique, it is expected that stable single-mode operation of verticalcavity surface-emitting lasers (VCSEL's) can also be enhanced

Manuscript received February 18, 1998; revised May 20, 1998. This work was supported by a HKU-CRCG grant and RGC grant.

The authors are with the Department of Electrical and Electronic Engineering, University of Hong Kong, Hong Kong.

Publisher Item Identifier S 1077-260X(98)06801-4. at high-power. In fact, it has been shown that VCSEL's with diffused QW structures exhibit stable fundamental mode operation at high power [4].

In this paper, a comprehensive numerical model to analyze the influence of interdiffusion on the steady static performance of VCSEL is presented. One major advantage of this model over the previous works [2]-[5] is the consideration of the implanted ions profile inside the laser cavity after rapid thermal annealing. Hence, the nonuniform distribution of electrical conductivity and absorption loss can be evaluated. The variation of optical gain and refractive index inside the QW active layer, arises from the interdiffusion, can also be calculated. In this model, the nonuniform distribution of: 1) temperature and voltage is solved by a finite-difference method; 2) optical field is calculated by beam propagation method such that the reflectivity of the Bragg reflector can be determined; and 3) carrier concentration along the QW active layer is also computed by a rate equation of carrier concentration. Using the above numerical techniques, the quasi-3-D distribution of temperature, voltage and optical field are computed in a self-consistent manner. This paper is organized as follows: In Section II, a self-consistent model of VCSEL with diffused QW structure is presented. In Section III, the steady-state characteristics of a gain-guiding VCSEL with diffused QW structure are analyzed. It can be shown that diffused QW structure enhances single-mode operation at high power. Brief discussion and conclusion are given in Section IV.

\section{LASER MODEL}

\section{A. Device Structures}

VCSEL with diffused QW structure under investigation is shown in Fig. 1. It is assumed that the laser has a circular metal contact of diameter $10 \mu \mathrm{m}$ on the expitaxial side (pside) for current injection. An active layer is sandwiched between two undoped spacer layers and two Bragg reflectors. The undoped spacer layers have thickness of half-wavelength each $(\sim 0.21 \mu \mathrm{m})$. The Bragg reflectors are formed by alternate layers of InP and InGaAsP with quarter-wavelength-thick dielectric layers on both the $\mathrm{n}$ - and $\mathrm{p}$-side, respectively. The total number of layers is equal to $20(\sim 2.1 \mu \mathrm{m})$ on the pside and $46(\sim 5.0 \mu \mathrm{m})$ on the $\mathrm{n}$-side. The active layer consists of six $\operatorname{In}_{0.53} \mathrm{Ga}_{0.47} \mathrm{As}-\mathrm{InP}$ QW with well width of $100 \AA$ and barrier thickness of $150 \AA$. It is assumed that the total thickness of the active layer is about half-wavelength $(\sim 0.2 \mu \mathrm{m})$. The lasing wavelength, $\lambda_{o}$, of the device is set to $1.55 \mu \mathrm{m}$. Light can be emitted from the n-Bragg reflector through a transparent 


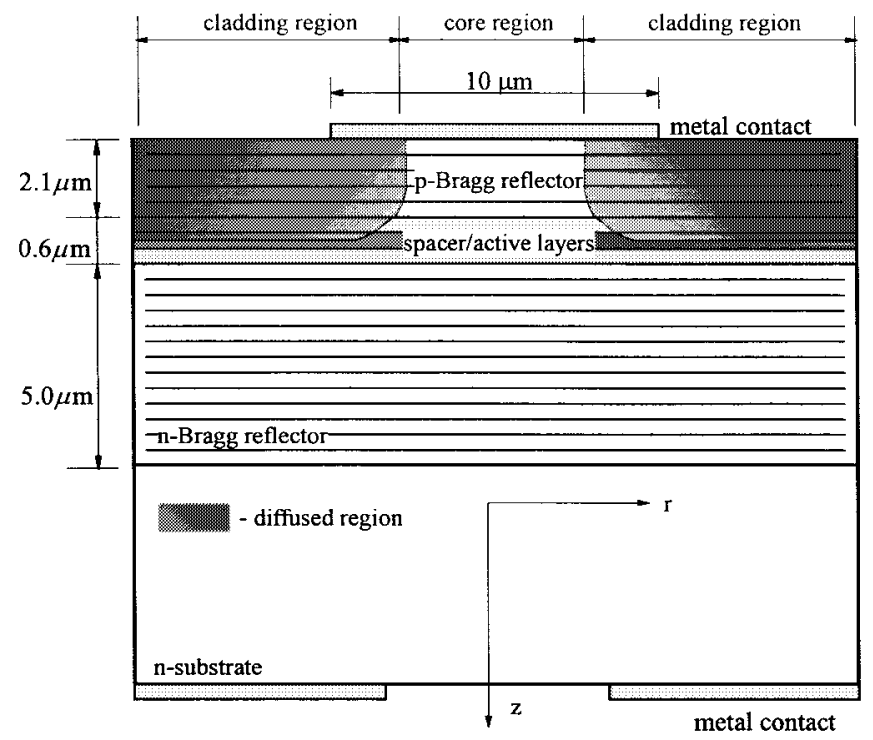

Fig. 1. Schematic diagram of a VCSEL with diffused QW structure. The diffused area forms the cladding region of the laser.

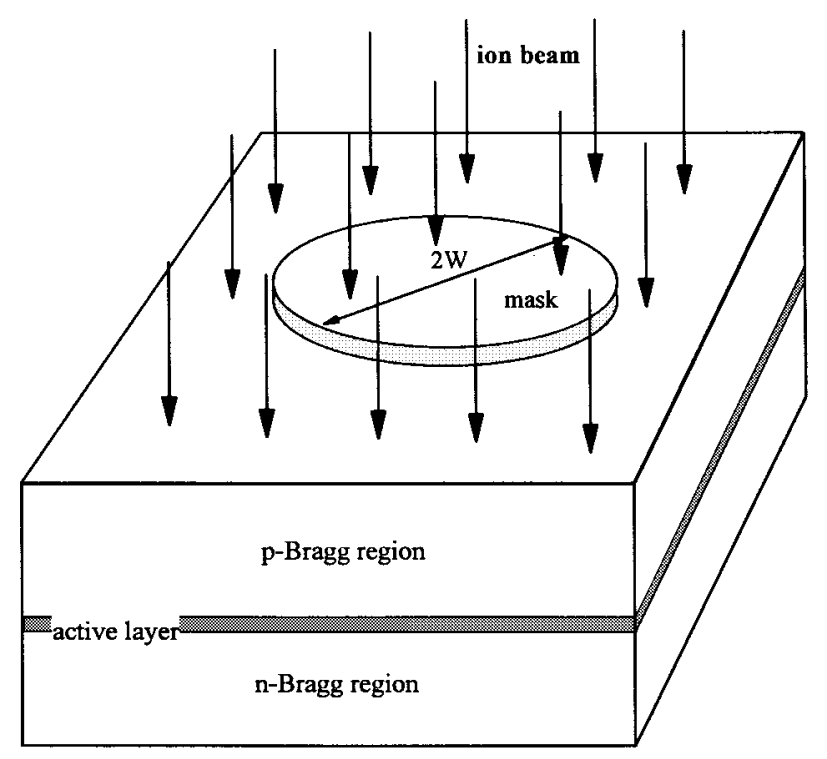

Fig. 2. Ion-implantation through the Bragg reflector into active layer. $W$ is the radius of the circular mask.

InP substrate. Alternatively, light can emit from the p-Bragg reflector through an indium-tin-oxide transparent electrode [6].

\section{B. Implantation and Thermal Annealing}

A circular mask of radius $W$ is used to cover the Bragg reflector on p-side, see Fig. 2. Ion beam of desired concentration and energy is bombarded into the unshielded region. It is assumed that the energetic ions penetrating into the semiconductor material will lose its energy through collisions with the target lattice and electrons and finally come to rest. Therefore, the depth of the penetration will dependent on the ion used, the kinetic energy of the ion as well as the structure of the target material. Using the approach given in [7], the distribution of ions $F(r, z)$ implanted through a circular mask is given by

$$
\begin{aligned}
F(r, z)= & \frac{N_{i o}}{\sqrt{2 \pi}\left\langle\Delta R_{p r}\right\rangle\left\langle\Delta R_{p z}\right\rangle} \exp \left\{-\frac{\left(z-\left\langle R_{p z}\right\rangle\right)^{2}}{2\left\langle\Delta R_{p z}\right\rangle^{2}}\right\} \\
& \times\left\{\frac{\left\langle\Delta R_{p r}\right\rangle}{2} \exp \left\{-\frac{(W-r)^{2}}{\left\langle\Delta R_{p r}\right\rangle^{2}}\right\}\right. \\
& \left.+\frac{\sqrt{\pi} r}{2} \operatorname{erfc}\left(\frac{W-r}{\left\langle\Delta R_{p r}\right\rangle}\right)\right\}
\end{aligned}
$$

where $\left\langle R_{p z}\right\rangle$ is the projection range, $\left\langle\Delta R_{p z}\right\rangle$ and $\left\langle\Delta R_{p r}\right\rangle$ are the vertical and transverse projected standard deviation of the implanted ion, respectively. The parameters $\left\langle R_{p z}\right\rangle,\left\langle\Delta R_{p z}\right\rangle$ and $\left\langle\Delta R_{p r}\right\rangle$ are the functions of implanting ions, implantation energy and substrate material. $N_{i o}$ is the implantation dose and $\operatorname{erfc}()$ is the complementary error function. After ionimplantation, diffusion of impurities takes place via rapid thermal annealing. The ions diffuse thermally into the active layer due to the concentration gradient. The annealing process can be described by the diffusion equation given as follows:

$$
\begin{aligned}
& \frac{\partial N_{i}(r, z, t)}{\partial t} \\
& =D_{\text {imp }}\left\{\frac{1}{r} \frac{\partial}{\partial r}\left(r \frac{\partial N_{i}(r, z, t)}{\partial r}\right)+\frac{\partial^{2} N_{i}(r, z, t)}{\partial z^{2}}\right\}
\end{aligned}
$$

where $N_{i}$ is the impurity concentration and $D_{\text {imp }}\left(=10^{-11}\right.$ $\mathrm{cm}^{2} \mathrm{~s}^{-1}$ ) is the diffusion coefficient of the impurity at a specific annealing temperature. It is assumed that no impurity is escaped from the device to the surrounding such that the corresponding boundary conditions are given by

1) $\frac{\partial N_{i}}{\partial r}=0$ at the edge of the device;

2) $\frac{\partial N_{i}}{\partial z}=0$ at the top and bottom surface of the device.

The nonuniform distribution of $N_{i}$ has significant influence on the p-side Bragg reflector region and QW active layer: 1) the destruction of lattice alters the electrical conductivity and optical absorption loss inside the p-side Bragg reflector, and 2 ) the impurities induced compositional disordering varies the indium content of the QW active layer.

In the ion-implanted region of the p-Bragg reflector; it is assumed that the electrical conductivity is dependent on the distribution of impurity concentration, $N_{i}(r, z)$. Therefore, the electrical conductivity of the ion-implanted area, $\sigma_{\text {imp }}$ $\left(\Omega^{-1} \mathrm{~cm}^{-1}\right)$, can be approximated by [8]

$$
\sigma_{\mathrm{imp}}(r, z) \approx \sigma_{o}(z)\left(1-\left(1-\frac{1}{K_{i}}\right) \frac{\Delta R_{p z} N_{i}(r, z)}{N_{i o}}\right)
$$

where $K_{i}\left(\sim 10^{6}\right)$ is the reduction factor. From (3), $\sigma_{\text {imp }}$ is a reducing function of $N_{i}$ due to the strength of lattice damage. Furthermore, the absorption loss, $\alpha\left(\mathrm{cm}^{-1}\right)$, due to the implanted ion inside the p-side Bragg reflector can also be approximated by

$$
\sigma(r, z) \approx \alpha_{\circ}\left(1-\gamma\left(1-\frac{1}{K_{i}}\right) \frac{\Delta R_{p z} N_{i}(r, z)}{N_{i o}}\right)^{-1}
$$

where $\alpha_{o}\left(=10 \mathrm{~cm}^{-1}\right)$ is the absorption loss without the implanted ion and $\gamma(=0.05)$ is a scaling factor. 
Inside the QW active layer, the diffusion coefficient of indium, $D_{\mathrm{In}}$, is proportional to the local impurity density $N_{i}$. The corresponding interdiffusion rate can be described by:

$$
D_{\text {In }}=\eta D_{\mathrm{imp}} N_{i}(r, z, t)
$$

where $\eta$ is a numerical factor. In our model, it is indium ions that diffuse from the barrier region, InP, into the quantum well, $\mathrm{In}_{0.53} \mathrm{Ga}_{0.47}$ As. Hence, the concentration profile of indium atoms, $C(r, z, t)$, after thermal annealing can be calculated from

$$
\begin{aligned}
\frac{\partial C(r, z, t)}{\partial t}= & D_{\operatorname{In}}\left\{\frac{\partial^{2} C}{\partial r^{2}}+\frac{\partial^{2} C}{\partial z^{2}}\right\} \\
& +\left\{\frac{\partial D_{\operatorname{In}}}{\partial r}\left(\frac{\partial C}{\partial r}\right)+\frac{\partial D_{\operatorname{In}}}{\partial z}\left(\frac{\partial C}{\partial z}\right)\right\}+\frac{D_{\operatorname{In}}}{r} \frac{\partial C}{\partial r}
\end{aligned}
$$

where the boundary conditions are the following:

1) $\frac{\partial C}{\partial r}=0$, at the edge of the device;

2) $D_{b} \frac{\partial C}{\partial r}=D_{\mathrm{qw}} \frac{\partial C}{\partial z}$, at the interfaces between barrier and well.

In the calculation, the initial concentration profile of indium atoms is assumed the same as that of the as-grown QW. In addition, the values of $D_{b}$ and $D_{\mathrm{qw}}$ are set to equal. If $N_{i}$ is assumed to be time independent, $D_{\mathrm{In}}$ and its derivatives can be determined and utilized as the constant coefficients of (6). Hence, $C$ in (6) can be solved numerically as if $N_{i}$ in (2).

The extent of interdiffusion inside the QW active layer is characterized by a diffusion length $L_{d}$ defined as

$$
\begin{aligned}
\int_{z-L_{z}}^{z+L_{z}} C\left(r, \xi, \tau_{a}\right) d \xi=w_{o} \int_{z-L_{z} / 2}^{z+L_{z} / 2} & \left(1-\left[\frac{1}{2} \operatorname{erf}\left(\frac{L_{z}+2 \xi}{4 L_{d}}\right)\right.\right. \\
+ & \left.\left.\operatorname{erf}\left(\frac{L_{z}-2 \xi}{4 L_{d}}\right)\right]\right) d \xi
\end{aligned}
$$

where $L_{z}$ is the well width, $w_{0}$ is the initial concentration of indium and $\tau_{a}$ is the annealing time. The influence of interdiffusion on the refractive index and optical gain of the QW active layer can also be calculated using the diffusion length $L_{d}$ as the parameter. Detailed calculation of the optical gain and the refractive index of the QW active layer under interdiffusion can be found in [9] and [10].

\section{Thermal Model}

The heat equation which governs the steady state heat flow in an axis-symmetric structure of VCSEL is given by

$$
\nabla \cdot\left(\lambda_{C}(r, z) \nabla T(r, z)\right)=-p(r, z)
$$

where $T$ is the absolute temperature in the laser, $\lambda_{C}$ $\left(\mathrm{W} \cdot \mathrm{cm}^{-1} \cdot \mathrm{K}^{-1}\right)$ is the thermal conductivity of the semiconductor material. It is assumed that the internal heat density $p(r, z)$ (in $\mathrm{W} \cdot \mathrm{cm}^{-3}$ ) is caused by 1 ) joule heating in both $\mathrm{p}$ - and $\mathrm{n}$-side Bragg reflectors and the spacer layers and 2) nonradiative recombination and spontaneous radiation inside the active layer. The expressions of different heat sources inside the laser cavity are given in the Appendix.
The corresponding boundary conditions of the laser are given as the following:

1) $T(r, 0)=T_{\mathrm{HS}}$ at the junction of the heatsink, where $T_{\mathrm{HS}}(=300 \mathrm{~K})$ is the temperature of heatsink;

2) $\frac{\partial T}{\partial_{r}}=\frac{\partial T}{\partial z}=0$, at the edge, top and bottom surface of the device.

The first boundary condition assumed that heat flows transversely to the heatsink with infinite capacity and the second condition specified that there is negligible thermal energy radiates from the surface of the device.

\section{Poisson and Carrier Rate Equations}

The behavior of carrier transport inside the Bragg, spacer and QW layers can be determined by self-consistent computation of Poisson equation and carrier rate equations over the laser cavity [11]. However, this numerical method requires extensive computational effort such that another numerical approach [12] is adopted in our calculation. This is done by solving 1) the Poisson equation over the Bragg and spacer layers for the nonuniform distribution of voltage and 2) the carrier rate equation of electrons over the QW active layer for the transverse distribution of electrons concentration. Therefore, the self-consistent solution for voltage and carrier concentration can be obtained by imposing the corresponding boundary conditions between the interfaces of spacer and QW active layers.

The Poisson equation used in this model is given by

$$
\nabla \cdot(\varepsilon(r, z) \nabla V(r, z))=Q(r, z)
$$

where $V(r, z)$ (Volt) is the potential distribution, $\varepsilon(\mathrm{F} / \mathrm{cm})$ is the permittivity of semiconductor material and $Q(r, z)\left(\mathrm{C} / \mathrm{cm}^{3}\right)$ is the charge density. $V(r, z)$ in (9) can be solved as if (8) by applying similar boundary conditions. It is noted that the Bragg reflectors are usually doped to reduce electrical resistance such that the external current injection has less influence on the distribution of electric field and the assumption of $Q(r, z)=0$ is roughly valid.

The carrier concentration, $N(r)$, along the transverse direction of the QW active region is described by

$$
\begin{aligned}
\frac{\partial N(r)}{\partial t}=\frac{J\left(z_{a}, r\right)}{q N_{z} L_{z}} & +D_{f} \frac{1}{r} \frac{\partial}{\partial r}\left(r \frac{\partial N(r)}{\partial r}\right) \\
& -R(N)-\nu_{g} g(N) \sum_{j}\left|E_{j}(r)\right|^{2}
\end{aligned}
$$

where $q$ is the electron charge, $N_{z}$ is the number and $L_{z}$ is the thickness of QW. $D_{f}\left(=10 \mathrm{~cm}^{2} \mathrm{~s}^{-1}\right)$ is the ambipolardiffusion coefficient, $\nu_{g}$ is the group velocity, $E$ is the transverse field distribution inside the active layer and $j$ is the mode number. $g(N)$ is the optical gain to be determined. The time dependent carrier-rate equation is solved subject to the conditions that $N$ and its derivative are continuous everywhere and at the axis of symmetry. The first derivative at $r=0$ take the form, $\left.\frac{\partial N}{\partial r}\right|_{r=0}=0$, due to the circular symmetry of the carrier concentration. It is also required that as the $r \rightarrow \infty$, $N \rightarrow 0$. The recombination loss of carrier concentration $R$ 
inside the active region is given by

$$
R(r)=\frac{N(r)}{\tau_{n}}+B N^{2}(r)+C_{\text {Aug }} N^{3}(r)
$$

where $\tau_{n}(=3 \mathrm{~ns})$ is the carrier lifetime, $B\left(=1 \times 10^{-10}\right.$ $\left.\mathrm{cm}^{3} \mathrm{~s}^{-1}\right)$ is the Bimolecular carrier recombination coefficient and $C_{\text {Aug }}\left(=3 \times 10^{-29} \mathrm{~cm}^{6} \mathrm{~s}^{-1}\right)$ is the Auger carrier recombination coefficient. The temperature dependence of the threshold current density $J_{\text {th }}$ at or above $300 \mathrm{~K}$ is described by the Arrhenius-type relation [13]

$$
J_{\mathrm{th}}=J_{o} \exp \left(\frac{T-300}{T_{o}}\right)
$$

where $J_{o}$ is the threshold current at $300 \mathrm{~K}$ and $T_{o}$ is the characteristic temperature. $J_{0}$ can be expressed as $J_{0}=$ $q N_{z} L_{z}\left(N_{\mathrm{th}} / \tau_{n}+B N_{\mathrm{th}}^{2}+C_{\mathrm{Aug}} N_{\mathrm{th}}^{3}\right)$ where $N_{\mathrm{th}}$ is the threshold carrier concentration at $300 \mathrm{~K}$.

Equations (9) and (10) are coupled via the voltage drop across the QW active region which can be approximated by

$$
\begin{gathered}
\frac{F_{e}(r)-F_{h}(r)}{q}=\frac{1}{q}\left[E_{g}+k_{B} T \cdot \ln \left\{\left(\exp \left(N(r) / N_{c}\right)-1\right)\right.\right. \\
\left.\left.\cdot\left(\exp \left(N(r) / N_{v}\right)-1\right)\right\}\right]
\end{gathered}
$$

where $E_{g}(=0.7199 \mathrm{eV})$ is the energy gap between the first quantized energy level of conduction and valence bands of $\mathrm{QW}$ and $k_{B}$ is the Boltzmann constant. $N_{c}$ and $N_{v}$ are the effective conduction and valence edge density of states, respectively, and can be expressed as

$$
N_{c / v}=m_{e / h}^{*} k_{B} T / \pi \hbar^{2} L_{z}
$$

where $m_{e / h}^{*}(=0.041 / 0.62)$ is the effective mass of electron/hole.

In (10), $J\left(z_{a}, r\right)$ represents the magnitude of injected current density at the interface of the $\mathrm{QW}$-active layer and is defined as

$$
J\left(z_{a}, r\right)=-\left.\sigma_{\text {act }}(r) \frac{\partial V(r, z)}{\partial z}\right|_{z=z_{a}}
$$

where $\sigma_{\text {act }}$ is the conductivity of the active layer and $z_{a}$ is the longitudinal position of the active layer.

\section{E. Optical Model}

The space-time evolution of the optical field, $E(r, z, t)$, inside the dielectric layers of VCSEL is governed by the wave equation given as

$$
\left(\frac{\partial^{2}}{\partial z^{2}}+\frac{\partial^{2}}{\partial r^{2}}+\frac{1}{r} \frac{\partial}{\partial r}\right) E=\frac{\varepsilon}{c^{2}} \frac{\partial^{2} E}{\partial t^{2}}+\frac{1}{c^{2}} \frac{\partial \varepsilon}{\partial t} \frac{\partial E}{\partial t}
$$

where $c$ is the velocity of light in free space and $\varepsilon$ is the complex dielectric constant of the laser medium. In (16), it is assumed that the optical field is uniform along the azimuthal direction. The optical field $E$ can be expressed as

$$
E(r, z, t)=\Psi^{+}(r, z, t) e^{i(\omega t-\beta z)}+\Psi^{-}(r, z, t) e^{i(\omega t+\beta z)}
$$

where $\Psi^{+}(r, z, t)$ and $\Psi^{-}(r, z, t)$ are the slowly varying envelope of the forward and reverse traveling waves, respectively, along the longitudinal direction. $\beta$ is the longitudinal propagation coefficient, $i(=\sqrt{ }-1)$ is a complex number, and $\omega$ is the lasing frequency.

The time dependent wave equations for the forward and reverse fields $\Psi^{+}(r, z, t)$ and $\Psi^{-}(r, z, t)$ can be obtained by substituting (17) into (16) and neglect the second derivative of $\Psi^{ \pm}(r, z, t)$ with respect to $z$ and $t$. The corresponding wave equations can be written as [14]

$$
\begin{aligned}
& \frac{1}{\nu_{g}} \frac{\partial \Psi^{ \pm}(r, z, t)}{\partial t} \pm \frac{\partial \Psi^{ \pm}(r, z, t)}{\partial z} \\
& \quad=\frac{i}{2 \beta}\left[\frac{1}{r} \frac{\partial}{\partial r}\left(r \frac{\partial}{\partial r}\right)+\Delta \varepsilon(r, z, t) k_{o}^{2}\right] \Psi^{ \pm}(r, z, t)+U_{\mathrm{sp}}^{ \pm}(t)
\end{aligned}
$$

where $k_{o}=\left(2 \pi / \lambda_{o}\right)$ is the wavevector. The group index, $n_{g}$, has an expression of $n_{g}^{2}=\varepsilon+\omega(\partial \varepsilon / \partial \omega)$ and (19), found at the bottom of the page. In (19), $\Delta n_{b}(r, z)$ is the difference of builtin refractive index distribution $n_{b}(r, z)$ of the device and $\Delta n$ is the change of refractive index inside the QW active layer. An extra term $U_{\mathrm{sp}}^{ \pm}$represents the generation of spontaneous field inside the active layer coupled to the longitudinal propagation waves.

The forward and reverse waves $\Psi^{+}(r, z, t)$ and $\Psi^{-}(r, z, t)$ propagating along the Bragg reflectors can be described by scattering matrix [14]. Consider the traveling waves at the boundary between two adjacent layers (see Fig. 3), the traveling waves reflected and transmitted at the boundary can be related by

$$
\left[\begin{array}{l}
\Psi_{r}^{+} \\
\Psi_{r}^{-}
\end{array}\right]=\left[\begin{array}{ll}
s_{11} & s_{12} \\
s_{21} & s_{22}
\end{array}\right]\left[\begin{array}{l}
\Psi_{i}^{+} \\
\Psi_{i}^{-}
\end{array}\right]
$$

where $\Psi_{i}^{+}, \Psi_{i}^{-}, \Psi_{r}^{+}$, and $\Psi_{r}^{-}$are the incident and reflected waves, respectively. The elements of the square matrix in (20) correspond to the transmission and reflection coefficients and these parameters are given as

$$
\begin{aligned}
& s_{11}=s_{22}=2 \sqrt{n e_{h} n e_{l}} /\left(n e_{h}+n e_{l}\right) \\
& s_{21}=-s_{12}=\left(n e_{h}-n e_{l} /\left(n e_{h}+n e_{l}\right)\right.
\end{aligned}
$$

where $n e_{h}$ and $n e_{l}$ are the corresponding effective refractive index of the index layers. Detailed implementation procedures of the above optical model can be found in [14].

$$
\Delta \varepsilon= \begin{cases}2 n_{b}(r, z)\left(\Delta n_{b}(r, z)+\Delta n(N)\right)+i(g(N)-\alpha) / k_{o}, & \text { active layer } \\ 2 n_{b}(r, z) \Delta n_{b}(r, z)-i \alpha / k_{o}, & \text { elsewhere }\end{cases}
$$




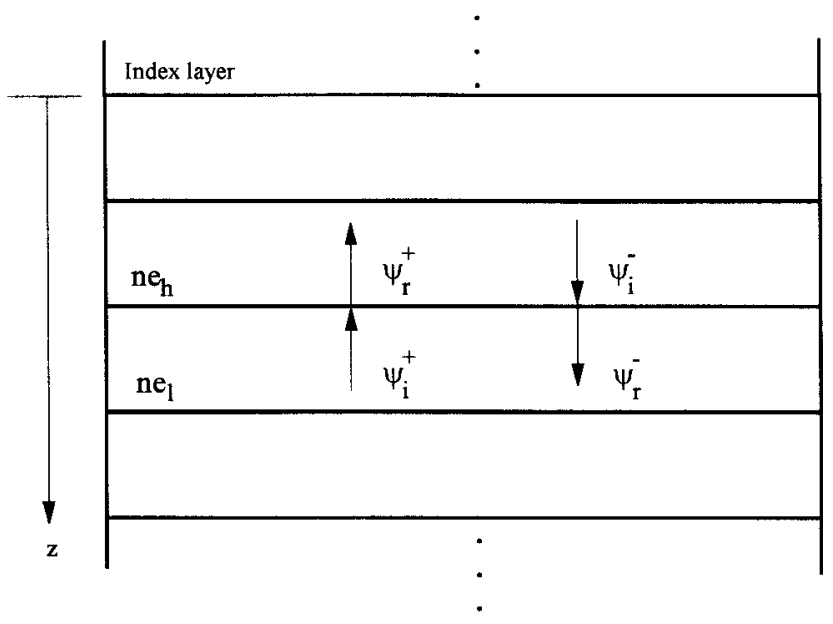

Fig. 3. Reflection and transmission of fields between boundary of index layers.

\section{F. Numerical Procedures}

In the above sections, all the equations required for the numerical analysis of VCSEL with a diffused QW structure have been derived. The flowchart for calculating the steady state behavior of VCSEL is shown in Fig. 4 and the operational principle of this model is outlined as follows.

1) At the beginning of the program, the quasi-3-D distribution of electrical conductivity and absorption loss inside the p-Bragg reflector as well as the refractive index and optical gain along the QW active layer are calculated under the influence of ion-implantation and rapid thermal annealing.

2) Using the information given in step 1), the quasi-3-D distribution of voltage inside the laser cavity and the nonuniform distribution of carrier concentration along the QW active layer are solved by the finite-difference method.

3) The quasi-3-D distribution of optical wave are computed using beam propagation method.

4) The quasi-3-D distribution of heat source and temperature are determined by the information of voltage distribution, carrier concentration and optical field profile obtained in the previous calculation.

5) It must be noted that the refractive index and optical gain are updated in each step between step 2) and step 4).

6) The above procedures, step 2) to step 5), are repeated until the convergence of voltage, carrier concentration, optical field and temperature is achieved.

\section{NUMERICAL ANALYSIS}

In the following calculations, the background electrical conductivity of the $n$-Bragg reflector, p-Bragg reflector, spacer/active layer and n-substrate are set to $1.5 \mathrm{~cm}^{-1} \cdot \Omega^{-1}$, $7 \mathrm{~cm}^{-1} \cdot \Omega^{-1}, 3 \mathrm{~cm}^{-1} \cdot \Omega^{-1}$ and $500 \mathrm{~cm}^{-1} \cdot \Omega^{-1}$, respectively. The background thermal conductivity of n-Bragg reflector, p-Bragg reflector and spacer/active layer are equal to 0.07 $\mathrm{W} \cdot \mathrm{cm}^{-1} \cdot \mathrm{K}^{-1}$, and that of $\mathrm{n}$-substrate is equal to 0.45 $\mathrm{W} \cdot \mathrm{cm}^{-1} \cdot \mathrm{K}^{-1}$, at $300 \mathrm{~K}$. In addition, their dependence on

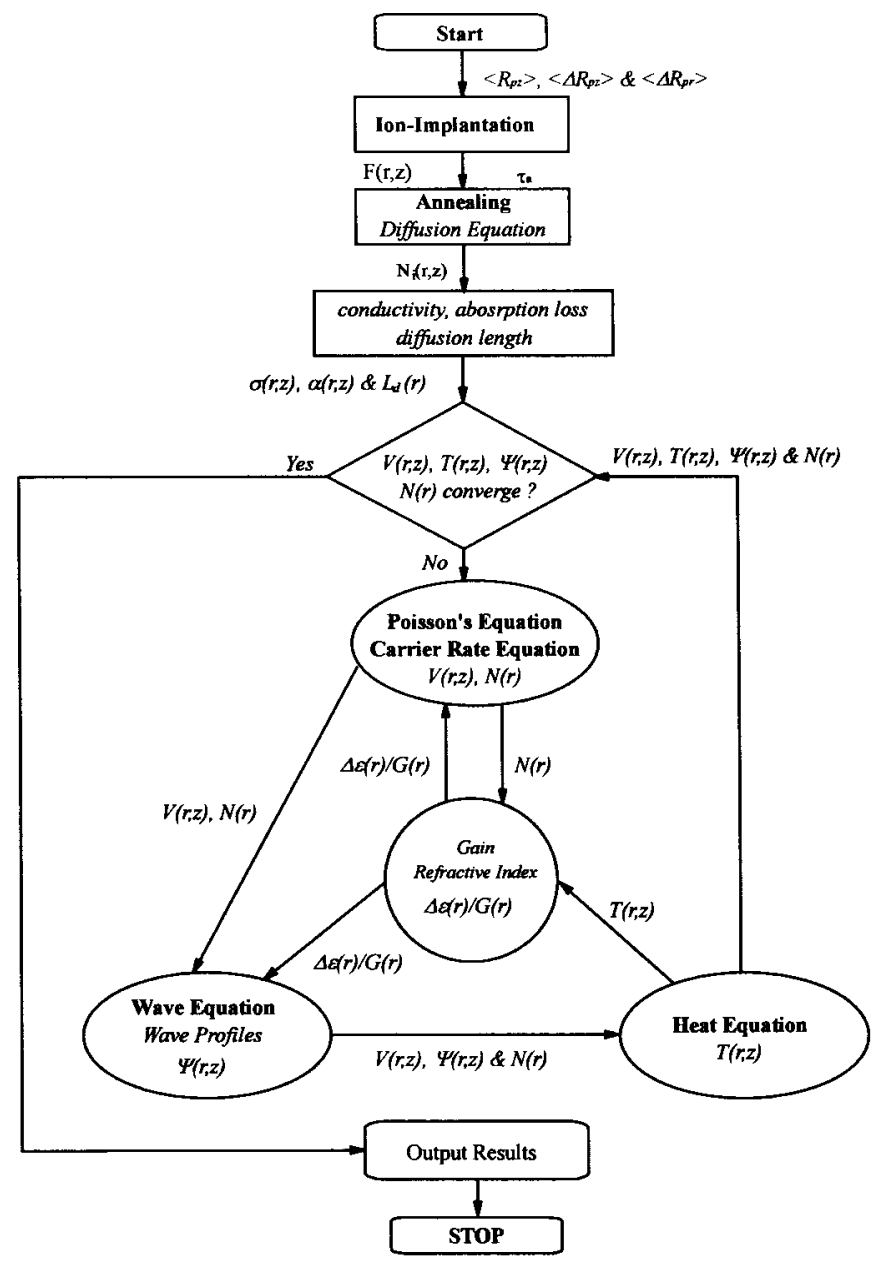

Fig. 4. Flowchart for the operational principle of the laser model.

temperature are assumed to be proportional to $T^{-1.2}$. It is assumed that the built-in refractive index of active layer, spacer layer, high index and low index of Bragg reflectors are equal to $3.524,3.393,3.503$ and 2.950 , respectively. The dependence of temperature and carrier concentration on the optical gain and refractive index of the diffused QW are calculated using the model similar to that given in [9] and [10].

Boron is adopted as the implanted impurity because the implantation of boron ions into $\operatorname{In}_{0.53} \mathrm{Ga}_{0.47} \mathrm{As}-\mathrm{InP} \mathrm{QW}$ increases the rate of diffusion on the group III sublattice. The enhancement of group III diffusion rate increases the indium content of the wells and its bandgap energy is decreased [15]. This implies that the refractive index of QW can be increased by interdiffusion technique. In the calculation, boron with concentration and energy of $10^{13} \mathrm{~cm}^{-2}$ and $240 \mathrm{keV}$, respectively, is penetrated into the $\mathrm{In}_{0.53} \mathrm{Ga}_{0.47} \mathrm{As}-\mathrm{InP} \mathrm{QW}$ active layer through the p-Bragg reflector. The parameters, $\left\langle R_{p z}\right\rangle,\left\langle\Delta R_{p z}\right\rangle$, and $\left\langle\Delta R_{p r}\right\rangle$, used in the calculation are equal to $0.54,0.193$, and $0.183 \mu \mathrm{m}$, respectively. Impurity induced compositional disordering is accomplished in the QW active layer by rapid thermal annealing at $500 \mathrm{~K}$ for about $30 \mathrm{~s}$. The implanted area of $\mathrm{p}$-Bragg reflector forms the cladding region and the diffused area of QW active layer establishes an antiguiding structure of the VCSEL. Fig. 5 shows the effective refractive index of spacer/active layers after ion-implantation 


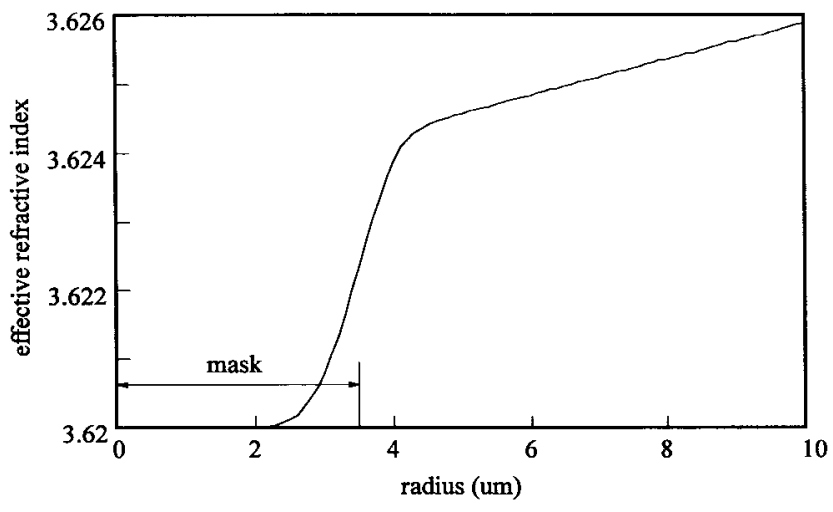

Fig. 5. Effective refractive index of spacer/active layers of VCSEL after implantation (using circular mask of $W=3.5 \mu \mathrm{m}$ ) and rapid thermal annealing.

(with circular mask of radius $W=3.5 \mu \mathrm{m}$ ) and rapid thermal annealing. It is observed that the refractive index increases near the cladding region but remains unchanged near the core region. It is expected that the influence of spatial hole burning and thermal lensing can be suppressed by the antiguiding structure such that single-mode operation is maintained at high power [4].

Fig. 6(a) and (b) shows the quasi-3-D distribution of electrical conductivity and absorption loss inside the p-Bragg region. Only half of the profile is shown in the diagram due to the symmetric distribution of the implanted ions. As we can see, the electrical conductivity decreases but the absorption loss increases near the cladding region of the pBragg reflector. The corresponding quasi-3-D distribution of voltage and temperature inside the laser cavity is shown in Fig. 7(a) and (b). Only half of the profile is shown in the diagram due to the symmetric distribution of voltage and temperature along the transverse direction. It is shown that the potential across the $\mathrm{QW}$ active layer is roughly equal to $0.8 \mathrm{~V}$. On the other hand, it is observed that the magnitude of temperature is maximum inside the core region of the $\mathrm{QW}$ active layer. This is because of the high joule heating and the nonradiative recombination spontaneous radiation occurred inside the QW active layer. The discontinuity of temperature distribution between $\mathrm{n}$-Bragg reflector and substrate is due to the large difference of thermal conductivity between these layers.

Fig. 8 shows the light output versus current $(L-I)$ curves of VCSEL with and without interdiffusion. It is observed that a kink occurs in the $L-I$ curves with the excitation of firstorder mode. The kink is shifted upward and stable fundamental mode operation can be maintained at a higher power level. The corresponding refractive index profile of VCSEL with and without interdiffusion operating at $1 \mathrm{~mW}$ is shown in Fig. 9. As we can see for the case without interdiffusion, the refractive index of the active layer increases near the center of the core. Due to the self-focusing effects, transverse modes move toward the center region and first order mode is excited. On the other hand, the increase in refractive index is counteracted by the diffused QW structure such that single-transverse-mode operation is maintained.

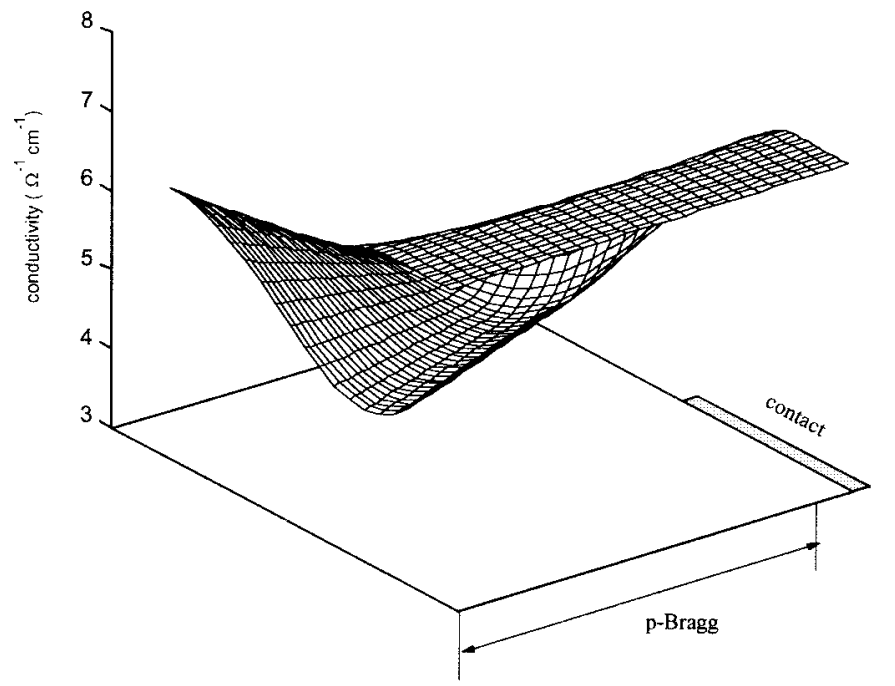

(a)

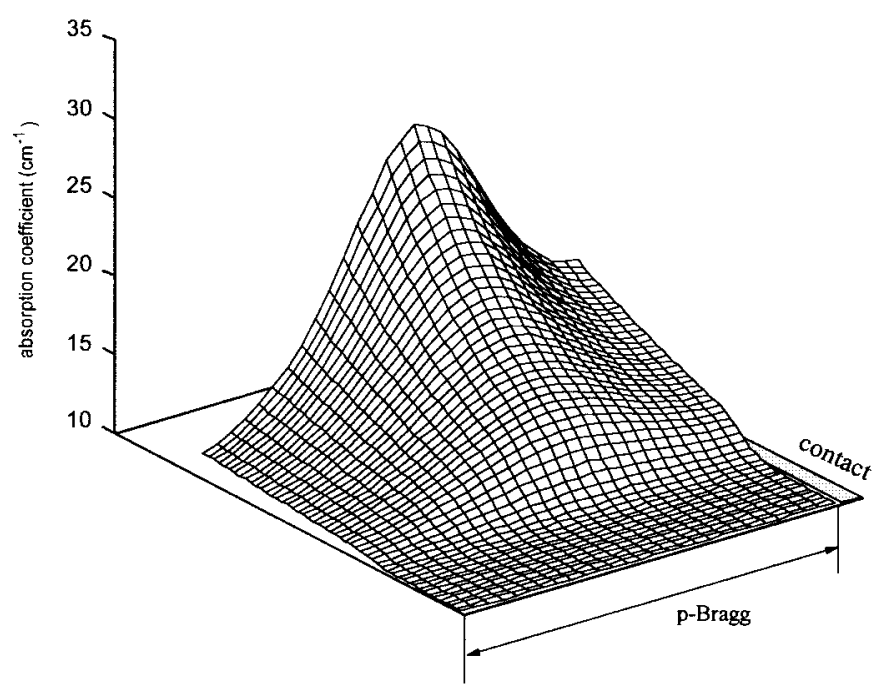

(b)

Fig. 6. The quasi-3-D distribution of (a) electrical conductivity and (b) absorption loss inside the p-Bragg reflector of VCSEL.

\section{DisCUSSION AND CONCLUSION}

The increase in refractive index inside the core region of QW active layer, arising from SHB and thermal lensing, excites high-order transverse modes in VCSEL. This is because the self-focusing effects collimate transverse modes into the core region of VCSEL such that the threshold gain of the transverse modes reduces. As a result, multiple transverse modes operation is observed in VCSEL at high power. In order to overcome the influence of self-focusing effects, gain-guided VCSEL with diffused QW structure is proposed. Due to the properties of interdiffusion using boron as the implanted ions, refractive index of $\operatorname{In}_{0.53} \mathrm{Ga}_{0.47} \mathrm{As}-\mathrm{InP} \mathrm{QW}$ can be increased such that an antiguiding structure is obtained against the influence of self-focusing effects. In addition, the current confinement along the transverse direction is improved due to the reduction of electrical conductivity inside the cladding region of the p-Bragg reflector. Other advantages of gainguided VCSEL with diffused QW structure over devices 


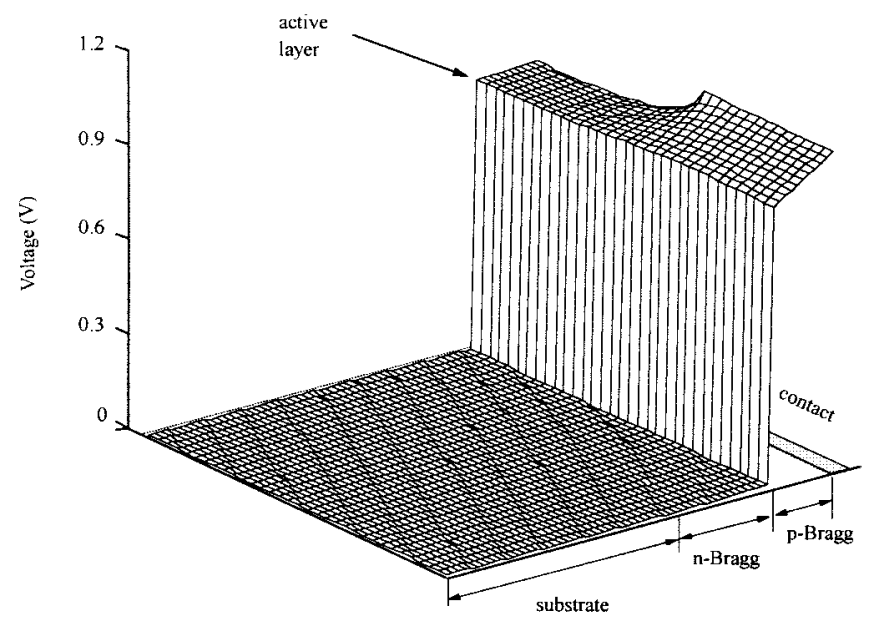

(a)

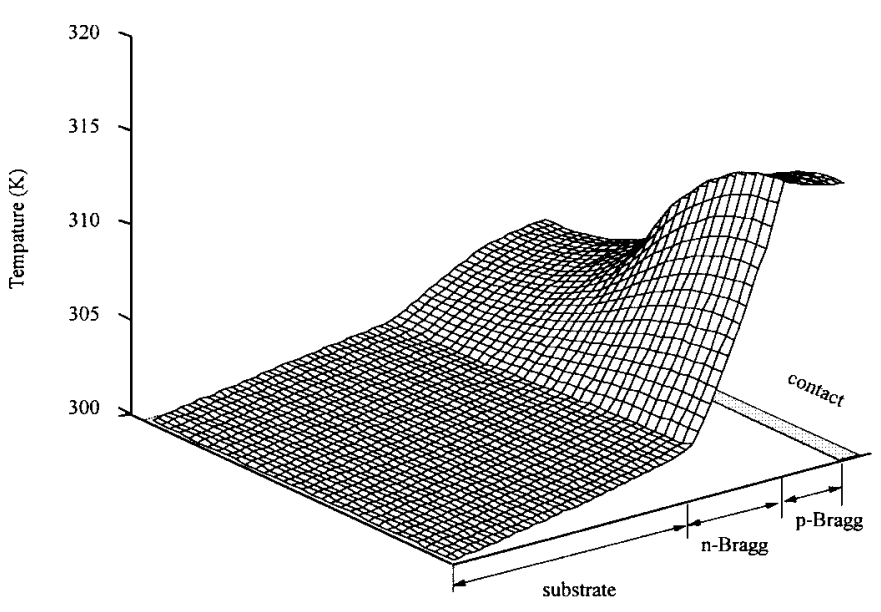

(b)

Fig. 7. The quasi-3-D distribution of (a) voltage and (b) temperature inside the laser cavity of VCSEL.

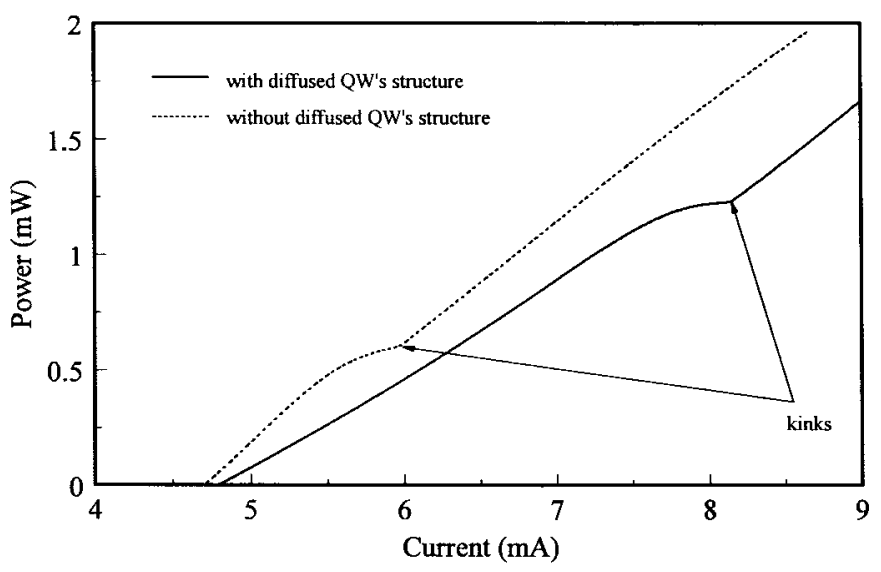

Fig. 8. $L-I$ curves of gain-guided VCSEL with (solid line) and without (dotted) diffused QW structure.

with passive antiguiding design [16] are 1) simple processing technique and 2) low production cost.

In conclusion, a quasi-3-D model of VCSEL with diffused QW structure is developed. The nonuniform distribution of electrical conductivity and absorption loss inside the p-Bragg reflector due to implanted ions are taken into consideration.

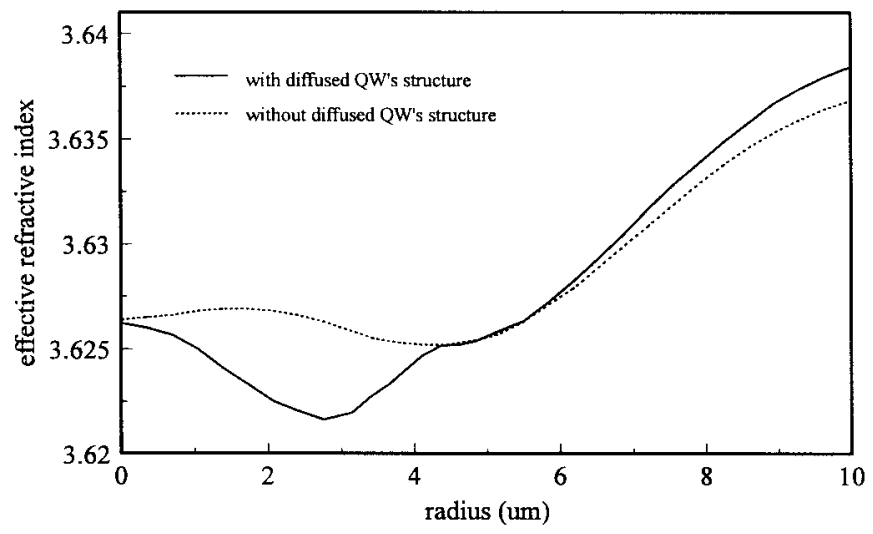

Fig. 9. Effective refractive index distribution of VCSEL with (solid line) and without (dotted) diffused QW structure.

The influence of impurity induced compositional disordering on the optical gain and refractive index of the QW active layer is also introduced into the model. Furthermore, the nonuniform distribution of temperature, voltage, and optical field is determined in a self-consistent manner. Using the model, the influence of implanted impurities on the steadystate characteristics of VCSEL is analyzed. It is shown that the single-mode operation of gain-guided VCSEL is enhanced by a diffused QW structure. The maximum output power for stable-fundamental-mode operation can be increased by double.

\section{APPENDIX}

For VCSEL with diffused QW structure, the electric conductivity is nonuniformly distributed. This is because of the continuous variation of electric conductivity due to the profile of ion-implantation. Furthermore, the heterojunction in the Bragg reflectors act as the anisotropic conductivity in the multilayer stacks. The specific resistance $R_{\text {het }, p}$ of the heterostructure in the p-type InP-InGaAsP Bragg reflectors is assumed equal to $2.5 \times 10^{-4} \Omega \cdot \mathrm{cm}^{2}$ and the resistance of the n-type heterojunction $R_{\text {het }, n}$ is approximated to be a factor of 10 lower than $R_{\text {het, } p}$ [8]. In our model, the total resistance of the Bragg reflectors is approximated by adding the resistivity of the heterojunction $R_{\text {het }}$ to the ohmic resistance, $\sigma$, of the dielectric layers [8].

The spatial variation of ohmic power loss per unit volume, $\mathrm{p}\left(\mathrm{W} / \mathrm{cm}^{3}\right)$, is given by [8]

$$
p(r, z)=\frac{J^{2}(r, z)}{\sigma(r, z)}
$$

where $J\left(\mathrm{~A} / \mathrm{cm}^{2}\right)$ is the current density and $\sigma\left(\Omega^{-1} \cdot \mathrm{cm}^{-1}\right)$ is defined as

$$
\sigma(r, z)= \begin{cases}\sigma_{\text {imp }}(r, z), & \text { implanted region } \\ \sigma_{o}(z), & \text { elsewhere }\end{cases}
$$

where $\sigma_{\text {imp }}(r, z)$ and $\sigma_{o}(z)$ are the region with and without ion-implantation, respectively. If a heterointerface is located in the cylinder segment of thickness $\Delta z$, we add the power density

$$
p(r, z)=\frac{R_{\mathrm{het}} J^{2}(r, z)}{\Delta z}
$$


to the ohmic loss. $R_{\text {het }}\left(\Omega \mathrm{cm}^{2}\right)$ is the resistivity of the heterojunction. The power dissipated inside the QW active layer, $p_{\text {act }}$, is described by [17]

$$
p_{\text {act }}(r)=R_{\text {act }} J_{\text {act }}^{2}(r) / L_{z}-S_{h v}(r)
$$

where $R_{\text {act }}\left(\Omega \cdot \mathrm{cm}^{2}\right)$ is the resistivity of the active layer and $S_{h v}\left(\mathrm{~W} \cdot \mathrm{cm}^{-3}\right)$ is the power density at active layer defined as

$$
S_{h v}(r)=\frac{1}{2} \nu_{g} h f S\left(z_{a}\right)\left|\Psi\left(r, z_{a}\right)\right|^{2} / L_{z}
$$

where $h(J-s)$ is the plank constant, $f$ is the operation frequency and $S\left(\mathrm{~cm}^{-3}\right)$ is the photon density at active layer.

\section{ACKNOWLEDGMENT}

The authors are grateful to Dr. E. H. Li, Department of Electrical and Electronic Engineering, University of Hong Kong, for useful discussion.

\section{REFERENCES}

[1] B. L. Weiss Ed., Special issue on quantum well mixing for optoelectronics, Opt. Quantum Electron., vol. 23, pp. S779-S994, 1991.

[2] S. F. Yu and E. H. Li, "Proposed enhancement of side mode suppression ratio in ?/4 shifted distributed feedback lasers with nonuniform diffused quantum wells," IEEE Photon. Technol. Lett., vol. 8, pp. 482-484, Apr. 1996.

[3] S. F. Yu, C. W. Lo, and E. H. Li, "Investigation of high power single mode operation in DFB and FP lasers using diffused quantum wells structure," IEEE J. Quantum Electron., vol. 33, pp. 999-1009, June 1997.

[4] S. F. Yu and P. C. Chui, "Proposed enhancement of single mode operation in VCSEL using diffused quantum wells structure," Opt. Quantum Electron., vol. 30, pp. 71-77, 1998.

[5] S. F. Yu, "Analysis and design of vertical cavity surface emitting lasers for self-sustained pulsation operation," IEEE J. Quantum Electron., vol. 34, pp. 497-505, Mar. 1998

[6] C. L. Chua, R. L. Thornton, D. W. Treat, V. K. Yang, and C. C. Dunnrowicz, "Indium tin oxide transparent electrodes for broad-area top-emitting vertical-cavity lasers fabricated using a single lithography step," IEEE Photon. Technol. Lett., vol. 9, pp. 551-553, May 1997.

[7] S. Furukawa, H. Matsumura, and H. Ishiwara, "Theoretical considerations on lateral spread of implanted ions," Jap. J. Appl. Phys., vol. 11, pp. 134-142, 1972.

[8] R. Michalzik and K. J. Ebeling, "Modeling and design of protonimplanted ultralow-threshold vertical cavity laser diodes," IEEE $J$. Quantum Electron., vol. 29, pp. 1963-1974, 1993.

[9] E. H. Li and K. S. Chan, "Laser gain and current density in a disordered AlGaAs/GaAs quantum well," Electron. Lett., vol. 29, pp. 1233-1234, 1993.

[10] E. H. Li, B. L. Weiss, K. S. Chan, and J. Micallef, "The polarization dependent refractive index of an interdiffusion induced AlGaAs/GaAs quantum well," Appl. Phys. Lett., vol. 62, pp. 550-552, 1992.

[11] Z. M. Li, M. Dion, S. P. McAlister, R. L. Williams, and G. C. Aers, "Incorporation of strain into a two-dimensional model of quantum well semiconductor lasers," IEEE J. Quantum Electronics, vol. 29, pp. 346-354, Feb. 1993.

[12] R. Papannareddy, W. Ferguson, and J. K. Butter, "Current spreading and carrier diffusion in zinc-diffused multiple-stripe-geometry lasers," Appl. Phys. Lett., vol. 50, no. 19, pp. 1316-1318, 1987.

[13] N. K. Dutta, J. Lopata, D. L. Sivco, and A. Y. Cho, "Temperature dependence of threshold of strained quantum well lasers," Appl. Phys. Lett., vol. 58, no. 11, pp. 1125-1127, 1991.

[14] S. F. Yu and C. W. Lo, "Influence of transverse modes on the dynamic response of vertical cavity surface emitting lasers," Proc. Inst. Elect. Eng.-J, Optoelectronics, vol. 143, no. 3, pp. 189-194, 1996.

[15] J. H. Marsh, S. A. Bradshaw, A. C. Bryce, R. Gwilliam, and R. W. Glew, "Impurity induced disordering of GaInAs quantum wells with barriers of AlGaInAs or GaInAsP," J. Electron. Mater., vol. 20, no. 12, pp. 973-978, 1991

[16] Y. A. Wu, G. S. Li, R. F. Nabiev, K. D. Choquette, C. Caneau, and C. J Chang-Hasnain, "Single-mode, passive antiguide vertical cavity surface emitting lasers," IEEE J. Select. Topics Quantum Electron., vol. 1, pp. 629-637, June 1995.

[17] S. F. Yu, W. N. Wong, P. Shum, and E. H. Li, "Theoretical analysis of modulation response and second harmonic distortion in vertical cavity surface emitting lasers," IEEE J. Quantum Electron., vol. 32 pp. 2139-2147, Dec. 1996.

W. M. Man, photograph and biography not available at the time of publication

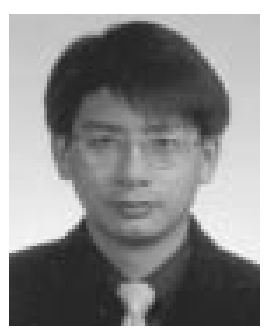

S.-F. Yu received the B.Eng. degree (with Departmental Prize) in electronic engineering from London University, University College, London, U.K., in 1990 and $\mathrm{Ph} . \mathrm{D}$. degree in optoelectronic from Cambridge University, Robinson College, Cambridge, U.K., in 1993.

He joined the Department of Electronic Engineering, Sha Tin Technical Institute, Hong Kong, as a part-time Lecturer in 1993. In 1994, he joined the Department of Electrical and Electronic Engineering, University of Hong Kong, where he was a Lecturer. Since 1996, he has been an Assistant Professor in the same department of the University of Hong Kong. His main research topics include wavelength selectivity of grating coupled waveguides, transient properties of semiconductor laser diodes, and design of optoelectronics integrated circuit. $\mathrm{He}$ currently conducts the development of high-performance semiconductor lasers using diffused quantum-well material for the application in high-speed communication systems. He also involves the investigation of optical soliton fiber communication system. He has published over 60 technical papers and one book chapter.

Dr. Yu is a Fellow and Honorary Scholar of Cambridge Commonwealth Trust Society. He held a Croucher Foundation scholarship and an overseas research student award while studying for the Doctoral program. His biography is published in the fourth edition of Who's Who in Science and Engineering (Marquis, USA), 25th edition of Dictionary of International Biography, and the 17th edition of International Directory of Distinguished Leadership. Dr. $\mathrm{Yu}$ is a member of the Optical Society of America. 